obstructive cardiomyopathy (HOCM). We have isolated troponin from HOCM muscle and studied its function using the in-vitro motility assay (IVMA). The level of troponin I (TnI) phosphorylation has been measured as $0.29 \pm 0.04 \mathrm{~mol} \mathrm{Pi} / \mathrm{mol}$ TnI compared with $1.62 \pm 0.06 \mathrm{~mol} \mathrm{Pi} / \mathrm{mol} \mathrm{TnI}$ in donor heart samples. The $\mathrm{Ca}^{2+}$-sensitivity of reconstituted thin filaments containing donor and HOCM troponin were measured. The HOCM troponin conferred a $\mathrm{Ca}^{2+}$-sensitivity that was not significantly different to that of the donor troponin. $\mathrm{EC}_{50} \mathrm{HOCM} /$ donor $=0.88 \pm 0.22(\mathrm{n}=8)$. HOCM troponin was treated with protein kinase A (PKA) (catalytic subunit) to increase the level of TnI phosphorylation to a similar level as found in donor hearts. There was no significant difference in the $\mathrm{Ca}^{2+}$ sensitivities of the thin filaments reconstituted with untreated or PKA-treated HOCM troponin. EC 50 HOCM/PKA treated HOCM=0.93 $\pm 0.32(n=4)$. Finally, donor troponin was dephosphorylated by treatment with acid phosphatase. In preliminary experiments this was compared with HOCM troponin, which has an intrinsically low level of phosphorylation. Thin filaments reconstituted with dephosphorylated donor troponin had a higher $\mathrm{Ca}^{2+}$ sensitivity than thin filaments containing HOCM troponin. Therefore, we conclude that HOCM troponin is modified in a manner independent of the causative mutation. HOCM troponin confers a $\mathrm{Ca}^{2+}$ sensitivity similar to troponin from donor hearts, which is independent of the phosphorylation status of TnI. This molecular phenotype is different from both acquired heart failure and genetic dilated cardiomyopathy.

\section{INVESTIGATING METABOLIC FLUX IN THE HYPERTHYROID HEART USING HYPERPOLARISED MAGNETIC RESONANCE}

doi:10.1136/hrt.2009.191064c

M Dodd, H Atherton, M Schroeder, L Heather, J Griffin, K Clarke, G Radda, D Tyler. Cardiac Metabolism Research Group, Department of Physiology, Anatomy and Genetics, University of Oxford, Oxford, UK

An increase in circulating thyroid hormone (TH) causes hypertrophy, which is accompanied by an increase in contractility and cardiac output. However, the mechanisms behind these observations are yet to be fully elucidated. TH is an important regulator of energy metabolism and has been shown to control the expression of many important lipolytic and glycolytic enzymes. One such enzyme is pyruvate dehydrogenase kinase, which, via phosphorylation, reduces the activity of pyruvate dehydrogenase $(\mathrm{PDH})$ and thus decreases the conversion of pyruvate to acetyl CoA, a key substrate in ATP synthesis. However, it is not known whether PDH activity is inhibited in the hyperthyroid rat heart. Hyperpolarised substrates increase the sensitivity of magnetic resonance spectroscopy (MRS) so that it is possible to investigate the flux of metabolites through specific enzymes in vivo. In this study, hyperpolarised pyruvate has been used in conjunction with MRS localised to the heart to monitor real time metabolic flux through $\mathrm{PDH}$ in hearts of control rats and rats injected with $\mathrm{TH}$ (triiodothyronine; T3) for 7 days ( $n=8$ per group). PDH activity measurements were made at baseline and after 7 days of injections. Hyperpolarised pyruvate was injected over $10 \mathrm{~s}$ into the anaesthetised rat via the tail vein. The conversion of pyruvate to alanine, lactate and bicarbonate was monitored every $1 \mathrm{~s}$ for 1 minute. The bicarbonate/pyruvate ratio was used as a measure of flux through $\mathrm{PDH}$. After 7 days administration of T3, flux through cardiac PDH was reduced by $76 \%(p<0.01)$. Thus, hyperpolarised pyruvate has revealed $\mathrm{PDH}$ inhibition to contribute to the pathology of the hyperthyroid heart.

\section{EVIDENCE FOR INWARD RECTIFIER POTASSIUM CHANNELS IN HL-1 CELLS}

doi:10.1136/hrt.2009.191064d

D Goldoni, A Collins. Centre for Vision and Vascular Science, School of Medicine and Dentistry, Queens University Belfast, Belfast, UK

Cardiac inward rectifier potassium current (IK1) plays a paramount role in repolarisation and stabilisation of the membrane potential in cardiomyocytes. Its downregulation contributes to arrhythmogenesis in heart failure and post-myocardial infarction. We have undertaken the molecular and functional characterisation of IK1 in the HL-1 cell line, which is derived from a mouse atrial tumour. HL-1 cells express many genes typical of differentiated adult cardiomyocytes and so far they are the only cardiac cell line able to divide continuously while maintaining a differentiated cardiac phenotype. ${ }^{1}$ IK1 has not been described in HL-1 cells. Whole-cell recording was performed under conditions that have been shown to preserve IK1 in excised membrane patches; the pipette solution contained $10 \mathrm{mM}$ pyrophosphate, $5 \mathrm{mM}$ fluoride and $0.1 \mathrm{mM}$ vanadate. Extracellular barium at an IK1-selective concentration $(0.1 \mathrm{mM}$ ) inhibited inward current by $44 \% \pm 4.2 \%$ (mean \pm SEM, $n=13$ cells), with little or no effect on outward current. The apparent reversal potential of the barium-sensitive current was $-58 \pm 6.0 \mathrm{mV}$ in $20 \mathrm{mM}$ and $-10 \pm 3.9 \mathrm{mV}$ in $100 \mathrm{mM}$ extracellular potassium with $160 \mathrm{mM}$ potassium in the pipette $(n=7)$. Reverse transcriptase PCR was performed with HL-1 complimentary DNA and primers specific for Kir2.1 (KCNJ2), the dominant IK1 isoform in cardiomyocytes. A PCR fragment of the expected size and sequence was produced. These results support the conclusion that HL-1 cells have an inwardly rectifying potassium current and express Kir2.1.

Acknowledgements The authors would like to thank Professor Barbara J McDermott for HL-1 cells and Dr You You Zhao for HL-1 cDNA.

Funding D Goldoni is supported by a studentship from the Harold McCauley Fund for Cardiovascular Research.

\section{REFERENCE}

1. Claycomb, et al. Proc Natl Acad Sci U S A 1998;95:2979-84.

\section{CONCENTRATION-DEPENDENT PROTECTION BY NO DONORS AGAINST ISCHAEMIA-REPERFUSION INJURY IN PERFUSED RAT HEARTS}

doi:10.1136/hrt.2009.191064e

W H Y Cheng, A P Halestrap, E J Griffiths. Department of Biochemistry and Bristol Heart Institute, University of Bristol, Bristol, UK

Nitric oxide (NO) has the potential to be beneficial in attenuating ischaemia-reperfusion (IR) injury, or deleterious due to its tendency to form reactive nitrogen species (RNS). In addition, different donors release varying amounts of $\mathrm{NO}$, making them difficult to compare directly. We studied the effects of two NO donors, SNAP (S-nitroso- $N$-acetyl-DL-penicillamine) and DEA/NO (diethylamine NONOate). Isolated rat hearts were perfused with or without SNAP at $2-100 \mu \mathrm{M}$ or DEA/NO at $2-20 \mu \mathrm{M}$. The NO donors were added 20 minutes before the onset of ischaemia ( 30 minutes) and remained present throughout reperfusion (60 minutes). Injury was assessed by measuring left ventricular developed pressure (LVDP) and lactate dehydrogenase $(\mathrm{LDH})$ release. SNAP significantly protected hearts from IR injury at 20 and $40 \mu \mathrm{M}$, but not $2 \mu \mathrm{M}$ or $100 \mu \mathrm{M}$, compared with controls, as indicated by improved recovery of LVDP and reduced $\mathrm{LDH}$ release ( $\mathrm{n}=6$ for each condition). Protection with DEA/ $\mathrm{NO}$ was seen at $2 \mu \mathrm{M}$ but at $20 \mu \mathrm{M}$ protection was lost. In conclusion, NO donors protected hearts, but the effect was concentration dependent; protection being lost at higher levels of the 